\title{
Kinetic control of the current through a single molecule
}

\author{
E.G. Petrov ${ }^{\text {a }}$, Ya.R. Zelinskyy ${ }^{\text {a }}$, V. May ${ }^{\text {b,* }}$, P. Hänggi ${ }^{c}$ \\ a Bogolyubov Institute for Theoretical Physics, National Academy of Sciences of Ukraine, Metrologichna str., 14-b, UA-03143 Kiev, Ukraine \\ ${ }^{\mathrm{b}}$ Humboldt-Universität zu Berlin, Institut für Physik - Newtonstraße 15, D-12489 Berlin, Germany \\ ${ }^{\mathrm{c}}$ Institut für Physik, Universität Augsburg, D-86135 Augsburg, Germany
}

Received 7 April 2006; accepted 28 June 2006

Available online 30 June 2006

\begin{abstract}
A unified description is put forward for the electron transmission through a molecule that is attached to two leads with the molecule being characterized by a single level to be populated by the transferred electrons. In deriving the expression for the current the Coulomb interaction is accounted for between the two extra electrons that may occupy the molecular level. The formation of two distinct transmission channels associated with the neutral and the singly charged molecule can directly be related to this interaction. Moreover, each transmission channel comprises a sequential as well as a direct (tunneling) pathway. The first pathway is realized via hopping transitions between the molecule and the neighboring electrodes. Just this inelastic kinetic process is responsible for the kinetic charging of the molecule. Then, the second pathway takes place against the background of kinetic molecular charging. In particular, it is demonstrated that hopping transmissions which are asymmetric with respect to the two electrodes cause a kinetic current rectification. The transient population of the molecule, realized by the transferred electrons, determines the rectification; the latter becomes rather large for resonant transmission.
\end{abstract}

(C) 2006 Elsevier B.V. All rights reserved.

Keywords: Kinetic equations; Charge transmission; Rectification; Coulomb interaction

\section{Introduction}

During recent years a number of different experiments have been described demonstrating strong nonlinear current-voltage $(I-V)$ characteristics of single organic molecules attached to nanoelectrodes [1-10]. These nonlinear $I-V$ characteristics are connected with such specific effects like current rectification and negative resistance. The mechanisms being responsible for nonlinear currents are rather well understood. Among others we mention here the influence of the spatial and energetic structure of the molecule, the type of molecule-electrode coupling, the effect of gating conditions, etc. In Ref. [11], for example, it could be demonstrated that a molecular wire which forms a single chemical bound to one of the metal electrodes and stays in mechanical contact with the other electrode may work as

\footnotetext{
* Corresponding author.

E-mail address: may@physik.hu-berlin.de (V. May).
}

a rectifier. By introducing particular models for the charge transmission through a single molecule the concrete effects leading to current nonlinearities can be identified.

It has been anticipated that within the description of charge transmission through the system "left electrodemolecule-right electrode" (LMR-system) the molecule has to be considered as an open quantum system with its discrete energy levels coupled to the continuum of conduction-band levels of the macroscopic electrodes and the multitude of molecular vibrations probably forming a heat bath. The Landauer approach considers the molecule as a center of elastic scattering processes for the electrons, moving directly from one lead to the other. Apparently, such a transmission can also be considered as an elastic tunneling process $[5,12,13]$. Recent extensions of the Landauer theory also include inelastic tunneling processes $[14,15]$. An approximate effective Landauer approach that accounts for inelastic phonon processes has been studied in Refs. $[16,17]$. This descriptions of the electron transmission 
through a single molecule reproduces the experimental findings rather satisfactory if the molecule is covalently linked to the leads (which is the case for some types of break junctions [18]). An agreement between measured data and calculations based on the Landauer approach is less satisfactory, however, for a weak (noncovalent) molecule-lead coupling [10]. The alternative of Landauer approach could be achieved in using the nonequilibrium Green function approach [19,20].

Yet another alternative is associated with the nonlinear kinetic theory which is based on nonequilibrium density matrix method. It offers a unified description of charge transmission and incorporates besides the elastic tunneling transmission route also an additional sequential route that is based on a hopping scheme [21,22]. During the (elastic or inelastic) tunneling processes the molecular orbitals (MOs) are not populated by the transferred electrons. Their population is only caused by sequential transfer [4,23-26], where the transferred electron jumps between the conduction band states of the lead and the molecular levels. In Ref. [21] we could demonstrate that tunneling and the sequential route of charge transmission yield additive contributions to the total interelectrode current provided the population of the molecule by the transferred electrons remains small. If this is not the case the Coulomb interaction between the transferred electrons influences the charge transmission [24,27-34]. In particular, this interaction causes specific transmission channels associated with the charge state of the molecule [22].

The goal of the present communication is to demonstrate in which way charging of the molecule (caused by the hopping processes in the LMR-system) as well as the Coulomb interaction (between the electrons captured by the molecule in the course of the transmission process) control the current formation. We demonstrate that such a control includes a kinetic opening and closing of tunneling transfer routes. All the results on the character of the tunneling and sequential transmission processes will be obtained in using a multi-electron kinetic theory. In doing so, it becomes possible to study the conditions and limitations of the Landauer scattering method. We prove the fact that the molecular charging essentially determines the resonant charge transmission, i.e. the tunneling current is strongly modified by hopping processes of the charge. Since just the forward and backward hopping transitions of the charge specify the transfer kinetics in the LMR-system one can allude to kinetic molecular charging. We also show that at definite conditions the same kinetic charging of the molecule becomes responsible for a specific kinetic rectification of the current.

\section{Model set-up and basic equations}

To elucidate the effect of the Coulomb interaction among the transferred electrons as well as of the molecule-lead coupling in controlling the total current through the molecule a particular model is put forward. It includes a single isolated molecular level and we shall neglect an electron-vibrational coupling (cf. Fig. 1). Such a simplified description has been also used by other authors before when studying the current formation through a quantum dot $[35,36]$ or a single molecule [31]. Our main objective for using such a simplified description is to derive analytic expressions for the current which can be directly compared with those following from the scattering approach. Corrections related to important electron-vibration interactions result in some fine structures in the $I-V$-curves $[16,19,20,22,26,37-40]$ and are not considered here further. Therefore, the derived analytic results describe the coarsened $I-V$ characteristics of the LMR-device with a single active MO. We also assume nonmagnetic leads, the absence of a magnetic field, and a zero-spin state for the neutral molecule. All these simplifications then allow us to use the electronic Hamiltonian of the LMR-system in the form

$$
\begin{aligned}
H_{\mathrm{LMR}}= & \sum_{r=\mathrm{L}, \mathrm{R}} \sum_{\mathbf{k}, \sigma}\left[E_{r \mathbf{k}} a_{r \mathbf{k} \sigma}^{+} a_{r \mathbf{k} \sigma}+V_{r \mathbf{k}} a_{r \mathbf{k} \sigma}^{+} c_{\sigma}+V_{r \mathbf{k}}^{*} c_{\sigma}^{+} a_{r \mathbf{k} \sigma}\right] \\
& +\sum_{\sigma}\left[\varepsilon(V)+U c_{-\sigma}^{+} c_{-\sigma}\right] c_{\sigma}^{+} c_{\sigma},
\end{aligned}
$$

which corresponds to the so-called Anderson's Hamiltonian [41]. $E_{r \mathbf{k}}$ is the energy of an electron with wave vector $\mathbf{k}$ in the conduction band of the $r$ th electrode, and $V_{r \mathbf{k}}$ defines the transfer coupling between the molecular level with energy $\varepsilon(V)$ and the $r \mathbf{k}$ th band state. The electron creation operators are denoted as $a_{r \mathbf{k} \sigma}^{+}$and the annihilation operators as $a_{r \mathbf{k} \sigma}$ (the electron spin is fixed by $\sigma$ ). Respective operators for the molecular level are given by $c_{\sigma}^{+}$and $c_{\sigma}$. The Coulomb interaction between two extra electrons in the molecular level is taken in the Hubbard form and is defined via the repulsion parameter $U$ [42]. Note that the Hamiltonian, Eq. (1) has been used by various authors to describe electron transfer through an isolated level of quantum dot or a molecule (see, e.g. Refs. $[28,43,44]$ ).

Based on the Green's function approach it has been shown in Ref. [43] that charge tunnelling through a particular molecular level strongly depends on the population of the other levels. An alternative description is based on a

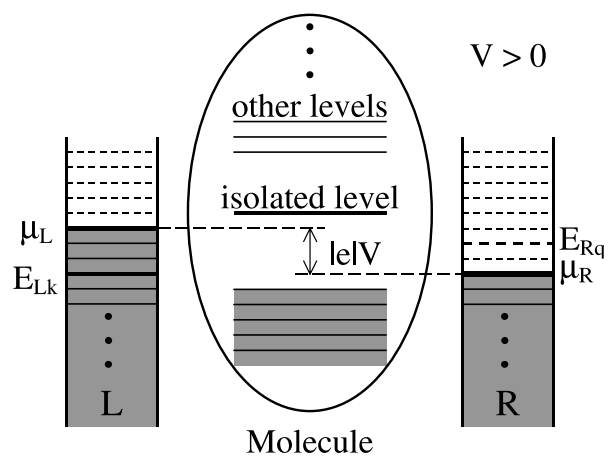

Fig. 1. Single-particle picture of the electronic levels in the considered LMR-system (molecular vibrations have been omitted). The LUMO level has been assumed to be separated from all other levels by a rather large energy gap (the chemical potential of the $r$ th electrode reads $\left.\mu_{r}=E_{\mathrm{F}}-|e| V_{r}, r=\mathrm{R}, \mathrm{L}\right)$. 
kinetic approach and directly computes the level occupation probabilities. It allows to analyze different nonlinear electron transfer effects associated with the interplay of molecular charging and an asymmetry of the metal-molecule coupling. For inelastic charge transfer, current peaks and a strong negative differential conductance have been predicted in Ref. [28]. Similar effects appear for sequential charge hopping along a molecular wire $[24,29]$. The interplay of Coulomb interaction and wire population within an incoherent charge transport regime has been studied in detail in Ref. [45].

In the present paper, sequential and tunneling contributions to an inter-electrode current are considered, also accounting for the Coulomb interaction between the electrons occupying the molecule in the course of their transmission. Therefore, charge transmission through the molecule is considered as a kinetic process in an open quantum system. A kinetic description supposes the presence of a dissipative environment coupled to the quantum system of interest. If molecular systems are considered the dissipative environment may be formed by inter- and intra-molecular vibrations (phonon reservoir). Neglecting any phonon contribution like in the Hamiltonian, Eq. (1) the dissipative environment is exclusively given by the macroscopic electrodes representing independent electronic reservoirs. Again, the molecule has to be considered as an open quantum system, and respective nonlinear kinetic equations can be used to calculate the electronic level populations $[22,46]$. Such a kinetic approach supposes that the width parameters $\Gamma_{\mathrm{L}}$ and $\Gamma_{\mathrm{R}}$ (see Eq. (11)) exceed the energies of those vibrations that are involved in the transmission process. (Any vibrational fine structure would be missing in the $I-V$ characteristics.)

Molecular $I-V$ characteristics depend strongly on the electrostatic profile in the inter-electrode region $[1,5,27$, 30]. In the most cases it is assumed that the voltage drop occurs only at the molecule-lead contacts, or the voltage is evenly distributed along the entire molecule. Generally, the profile is defined by the distribution of the electronic charge density within the molecule (see the calculations in Refs. [34,47,48]). Analytic considerations of this problem carried out in the framework of a tight-binding approach indicate that the electrostatic drop across the molecule strongly depends on the character of electron delocalization across those MOs which participate in the electron transmission [49]. If strongly delocalized MOs are involved in the transfer process, the voltage profile is not changed across the inner part of the molecule. Thus, the main voltage drop is biased at the molecule-lead contacts as well as the terminal molecular sites. If the localized MOs are responsible for the transmission, the voltage drop covers the whole molecule. In what follows we consider the transmission through a single localized MO. In this case, the electrostatic profile is changed along the whole LMRdevice except a small region of electron localization within the molecule. It means that the energetic shift of the MO is defined by a voltage division factor $\eta[1,5,49]$ and thus
$\varepsilon(V)=\varepsilon_{0}+\eta e V$,

where $\varepsilon_{0}$ is the position of the single-electron level at the absence of an applied voltage, and $V=V_{\mathrm{R}}-V_{\mathrm{L}}$ denotes the voltage bias between the right and the left electrode.

The current can be deduced from the following standard expression

$I=e \dot{N}_{\mathrm{L}}$

where $\dot{N}_{\mathrm{L}}\left(=-\dot{N}_{\mathrm{R}}\right)$ is the time-derivative of the total number of electrons present in the left (right) lead. Since the quantity

$N_{\mathrm{L}}(t)=\sum_{\mathbf{k} \sigma} P_{\mathrm{L} \mathbf{k} \sigma}(t)$

is defined by the (single-electron state) population $P_{\mathbf{L k} \sigma}(t)$ one has to derive a kinetic equation for this quantity. According to the hopping processes the molecular level is populated and the molecule may become singly or doubly charged. The respective molecular occupation probabilities are denoted as $W_{n}(t)$ where $n=0,1,2$ gives the number of excess electrons present at the molecule. Let $P_{\sigma}(t)$ be the single-electron population of the MO. This value coincides with the probability to find an extra electron with spin projection $\sigma$ at the MO. Then, the $W_{n}(t)$ can be expressed by the $P_{\sigma}(t)$ according to

$W_{0}(t)=\left(1-P_{\uparrow}(t)\right)\left(1-P_{\downarrow}(t)\right), W_{1 \uparrow}(t)=P_{\uparrow}(t)\left(1-P_{\downarrow}(t)\right)$,

$W_{1 \downarrow}(t)=\left(1-P_{\uparrow}(t)\right) P_{\downarrow}(t), W_{2}(t)=P_{\uparrow}(t) P_{\downarrow}(t)$.

Note the validity of $W_{0}(t)+W_{1 \uparrow}(t)+W_{1 \downarrow}(t)+W_{2}(t)=1$, which guarantees the conservation of the probability.

The kinetic equations for the single-electron populations $P_{\mathrm{Lk} \sigma}(t)$ and $P_{\sigma}(t)$ may be derived from a general nonlinear kinetic equation for the single-electron distribution function derived in Ref. [22], Eq. (38). Specified to the present case and, observing Eq. (4), one obtains

$\dot{N}_{\mathrm{L}}(t)=\dot{N}_{\mathrm{L}}^{(\mathrm{seq})}(t)+\dot{N}_{\mathrm{L}}^{(\mathrm{dir})}(t)$.

Physically, this value determines a total net charge flow from the left electrode to the right electrode. The first contribution on the right-hand side

$$
\begin{aligned}
\dot{N}_{\mathrm{L}}^{(\text {seq })}(t)= & -2\left[\chi_{\mathrm{L}}(0) W_{0}(t)-\chi_{-\mathrm{L}}(0)\left(W_{1 \uparrow}(t)+W_{1 \downarrow}(t)\right) / 2\right] \\
& -2\left[\chi_{\mathrm{L}}(1)\left(W_{1 \uparrow}(t)+W_{1 \downarrow}(t)\right) / 2-\chi_{-\mathrm{L}}(1) W_{2}(t)\right]
\end{aligned}
$$

results from the sequential charge transmission process. The rates $\chi_{\mathrm{L}}(j)\left(\chi_{\mathrm{R}}(j)\right)$ describe single-electron hopping transitions from the left (right) electrode into the molecule being in its ground (charge neutral) state $(j=0)$ or its singly charged state $(j=1)$. Rates of the reverse processes are denoted as $\chi_{-\mathrm{L}}(j)\left(\chi_{-\mathrm{R}}(j)\right)$. The second contribution on the right-hand side of Eq. (6), i.e.,

$\dot{N}_{\mathrm{L}}^{(\mathrm{dir})}(t)=-2\left[Q_{\mathrm{LR}}(0) W_{0}(t)+Q_{\mathrm{LR}}(1)\left(W_{1 \uparrow}(t)+W_{1 \downarrow}(t)\right)\right]$

describes a direct inter-electrode charge transmission. Here, the quantities $Q_{\mathrm{LR}}(0)$ and $Q_{\mathrm{LR}}(1)$ are the tunneling flows characterizing a left-to-right inter-electrode charge transmission if the molecule is in its neutral and its singly 
charged state, respectively. The weight of each flow component in a common tunneling flow (8) is determined by respective probabilities $W_{n}(t)$ to find the molecule in a particular charged state. In turn, each state is characterized by the single-electron population $P_{\sigma}(t)$ (cf. Eq. (5)) which obeys the following nonlinear kinetic equation

$$
\begin{aligned}
\dot{P}_{\sigma}(t)= & -\left[\left(\chi_{-\mathrm{L}}(0)+\chi_{-\mathrm{R}}(0)\right) W_{1 \sigma}(t)-\left(\chi_{\mathrm{L}}(0)+\chi_{\mathrm{R}}(0)\right) W_{0}(t)\right] \\
& -\left[\left(\chi_{-\mathrm{L}}(1)+\chi_{-\mathrm{R}}(1)\right) W_{2}(t)-\left(\chi_{\mathrm{L}}(1)+\chi_{\mathrm{R}}(1)\right) W_{1-\sigma}(t)\right] .
\end{aligned}
$$

It describes the temporal evolution of molecular charging and discharge in the course of the transmission process. Note that this evolution is characterized by the same rate constants $\chi_{\mathrm{L}(\mathrm{R})}(j)$ and $\chi_{-\mathrm{L}(-\mathrm{R})}(j)$ that also determine the hopping kinetics in the LMR-system. Therefore, the hopping processes are not only responsible for the formation of the sequential part (7) of the total charge flow (6) but also for the kinetic charging of the molecule. So, we may state that the kinetic molecular charging caused by inelastic hopping processes governs the tunneling current component in changing the probabilities $W_{0}$ and $W_{1 \uparrow}+W_{1 \downarrow}$, which characterize the occupation of the transient charging states of the molecule. This is a basically new result follow-

\section{$1^{\text {st }}$ transmission channel}
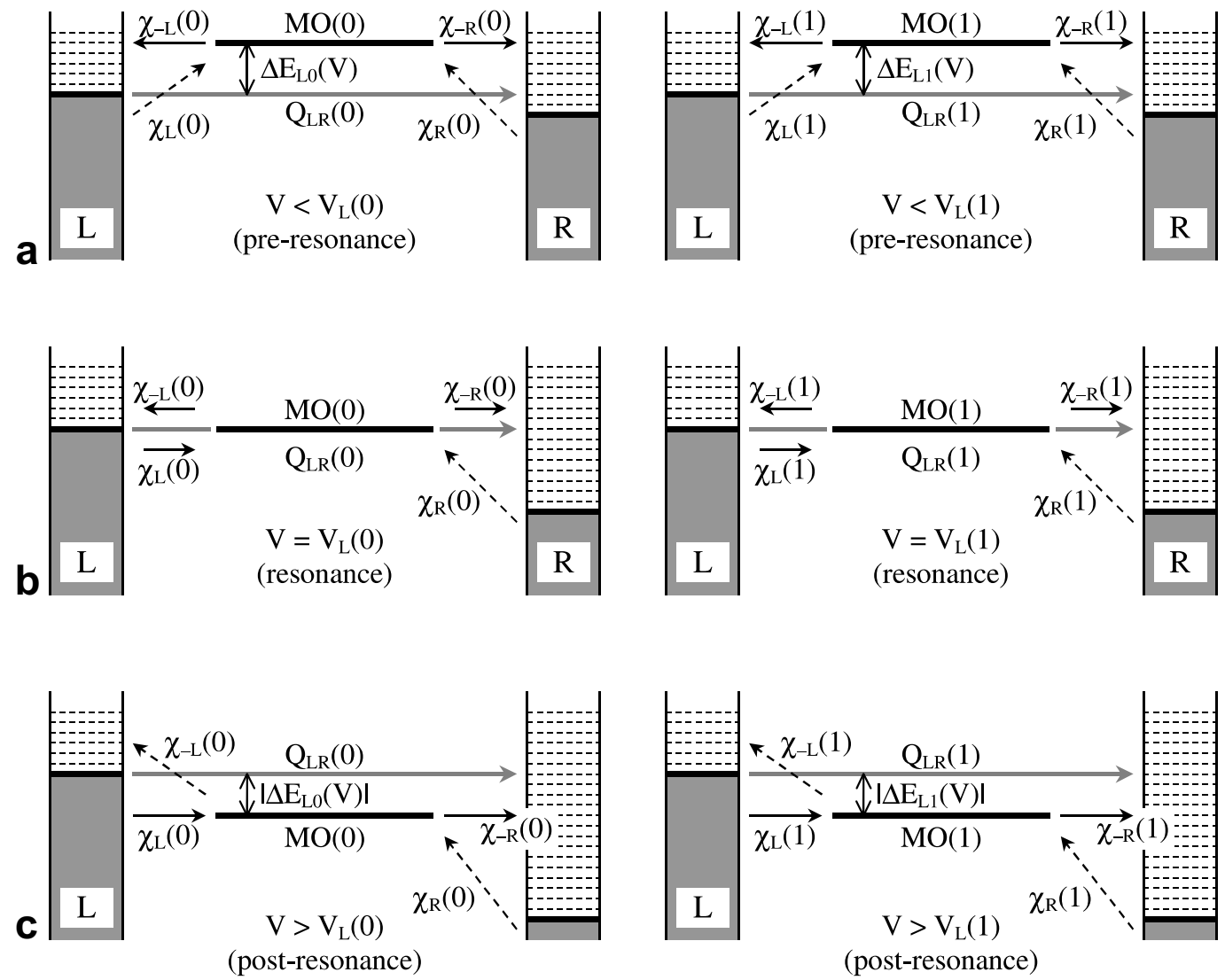

ing from the unified description of charge transmission through a molecule. It follows from the Eqs. (7) and (8) indicating that two different transmission channels are formed in the LMR-device despite the fact that only a single MO participates in the charge transmission. The first channel is opened with probability $W_{0}$ while the second one with probability $W_{1 \uparrow}+W_{1 \downarrow}$.

Fig. 2 shows the kinetic scheme of electron transmission through the LMR-system along with both transmission channels. Molecular charging is achieved by the jump of an electron from the conduction band of an electrode to the unoccupied or singly occupied $\mathrm{MO}$ (denoted as $\mathrm{MO}(0)$ or $\mathrm{MO}(1)$, respectively). The efficiency of the $\mathrm{MO}(0) \rightarrow$ $\mathrm{MO}(1)$ and $\mathrm{MO}(1) \rightarrow \mathrm{MO}(2)$ charging processes is characterized by the electrode-molecule rate constants $\chi_{\mathrm{L}(\mathrm{R})}(0)$ and $\chi_{\mathrm{L}(\mathrm{R})}(1)$, respectively. The reverse transfer processes $\mathrm{MO}(1) \rightarrow \mathrm{MO}(0)$ and $\mathrm{MO}(2) \rightarrow \mathrm{MO}(1)$ lead to a molecular discharge. Now, the jump of an electron from the singly and doubly occupied MO to the empty levels of the conduction band are described by respective molecule-electrode rate constants $\chi_{-\mathrm{L}(-\mathrm{R})}(0)$ and $\chi_{-\mathrm{L}(-\mathrm{R})}(1)$.

In the case of a tunneling process, the flows $Q_{\mathrm{LR}}(0)$ and $Q_{\mathrm{LR}}(1)$ characterize a distant unistep jump of an electron from one lead to another. The jump is influenced by the

Fig. 2. Kinetic scheme of a charge transfer along two transmission channels in the case of a single MO and at finite voltage $V>0$. The dashed arrows depict the noneffective hopping events. The long shadow arrows indicate the tunneling transfers formed with participation of virtual electronic states of the molecule. 
molecular charge state and, thus, depends on the concrete type of transmission channel. In contrast to electron hopping, the transferred electron only virtually occupies the MO during the tunneling process. (In the mesoscopic physics a similar type of tunneling transmission is known as a co-tunneling [50,51].) If a single frontier MO participates in the transmission, the virtual states are formed at the empty MO or at the MO already occupied by a single extra electron. Let the MO be empty and, thus, let charge transmission occur through a neutral molecule. In the pre-resonant region $V<V_{\mathrm{L}}(0)$ (scheme (a) in Fig. 2), the forward (electrode-molecule) hopping rates $\chi_{\mathrm{L}}(0)$ and $\chi_{\mathrm{R}}(0)$ are much smaller than the backward (molecule-electrode) hopping rates $\chi_{-L}(0)$ and $\chi_{-R}(0)$. Therefore, the (kinetic) population of the MO by an extra (transferred) electron is small. If the $\mathrm{MO}$ is in resonance with the Fermi-level of the left electrode (scheme (b) in Fig. 2) its population suddenly increases. It is defined by the relation between the rates $\chi_{\mathrm{L}}(0)\left(=\chi_{-\mathrm{L}}(0)\right)$ and $\chi_{-\mathrm{R}}(0)$. In the post-resonant region $V>V_{\mathrm{L}}(0)$ (scheme (c) in Fig. 2), the MO-population is defined by the relation between the rate constants $\chi_{\mathrm{L}}(0)$ and $\chi_{-\mathrm{R}}(0)$. Note that electron tunneling between the electrodes does not alter the charge state of the molecule. Consequently, the charge flow $Q_{\mathrm{LR}}(0)$ occurs with the participation of the unoccupied MO. Similar transfer processes appear for charge transmission along the second channel where the transmission takes place through a singly occupied MO. The difference of charge transfer along the two transmission channels is related to the single-electron gaps, Eq. (14). Because $\Delta E_{\mathrm{L}(\mathrm{R}) 1}(V)=\Delta E_{\mathrm{L}(\mathrm{R}) 0}(V)+U$ then the resonant regime of charge transmission along the second channel is switched on at a higher applied voltages $V_{\mathrm{L}}(1)=V_{\mathrm{L}}(0)+U / \eta|e|$ and $V_{\mathrm{R}}(1)=V_{\mathrm{R}}(0)+U /(1-\eta)|e|$ (cf. Eqs. (23) and (24)).

The hopping rates which appear in Eqs. (7) and (9) read $(r=\mathrm{L}, \mathrm{R})$

$$
\begin{aligned}
& \chi_{r}(j)=\frac{1}{\hbar} \int_{-\infty}^{+\infty} \mathrm{d} E \Gamma_{r}(E) f_{r}\left(E-e V_{r}\right) \delta(E-\varepsilon(V)-j U), \\
& \chi_{-r}(j)=\frac{1}{\hbar} \int_{-\infty}^{+\infty} \mathrm{d} E \Gamma_{r}(E)\left(1-f_{r}\left(E-e V_{r}\right)\right) \delta(E-\varepsilon(V)-j U) .
\end{aligned}
$$

$f_{r}(\epsilon)=\left\{\exp \left[\left(\epsilon-E_{r \mathrm{~F}}\right) / k_{\mathrm{B}} T\right]+1\right\}^{-1}$ denotes the Fermi distribution of the $r$ th electrode ( $E_{r \mathrm{~F}}$ is the respective Fermi-energy). Below, for the sake of clarity, we suppose that the left electrode is grounded and thus $V_{\mathrm{L}}=0, V_{\mathrm{R}}=V$. Furthermore, we set $E_{\mathrm{LF}}=E_{\mathrm{RF}}=E_{\mathrm{F}}$. The quantities

$\Gamma_{r}(E)=2 \pi \sum_{\mathbf{k}}\left|V_{r \mathbf{k}}\right|^{2} \delta\left(E_{r \mathbf{k}}-E\right)$

define the molecular level-broadening $\left(\Gamma_{\mathrm{L}}(E)+\Gamma_{\mathrm{R}}(E)\right) / 2$. They are caused by the molecule-lead coupling and may be deduced from the molecular self-energies $\Sigma_{\mathrm{L}}(E)$ and $\Sigma_{\mathrm{R}}(E)$ by the relation $\Gamma_{\mathrm{L}(\mathrm{R})}(E)=2 \operatorname{Im} \Sigma_{\mathrm{L}(\mathrm{R})}(E)$. If the so-called wide-band approximation can be used their dependence on the transmission energy $E$ remains weak
$[1,5,7,13]$. Thus, setting $\Gamma_{r}(E) \approx \Gamma_{r}$ one arrives at the following expressions for the hopping rates

$\chi_{r}(j) \approx(1 / \hbar) \Gamma_{r} n\left(\Delta E_{r j}(V)\right)$,

$\chi_{-r}(j) \approx(1 / \hbar) \Gamma_{r}\left(1-n\left(\Delta E_{r j}(V)\right)\right.$.

The quantity

$n\left(\Delta E_{r j}(V)\right)=\left[1+\mathrm{e}^{\Delta E_{r j}(V) / k_{\mathrm{B}} T}\right]^{-1}$

gives the electronic distribution function with the voltage controlled transmission gaps (cf. Fig. 3)

$\Delta E_{\mathrm{L} j}(V)=\Delta E(0)+j U+\eta e V$

$\Delta E_{\mathrm{R} j}(V)=\Delta E(0)+j U-(1-\eta) e V$.

$\Delta E(0) \equiv \varepsilon_{0}-E_{\mathrm{F}}$ denotes the unbiased energy gap between the molecular level and the Fermi energy. The gaps $\Delta E_{\mathrm{L} 0}(V)$ and $\Delta E_{\mathrm{R} 0}(V)$ refer to the transmission along the first channel (cf. the kinetic scheme in Fig. 2) while the gaps $\Delta E_{\mathrm{L} 1}(V)$ and $\Delta E_{\mathrm{R} 1}(V)$ are associated with the second transmission channel. Note that the gaps $\Delta E_{\mathrm{L} 1}(V)$ and $\Delta E_{\mathrm{R} 1}(V)$ exceed the respective gaps $\Delta E_{\mathrm{L} 0}(V)$ and $\Delta E_{\mathrm{R} 0}(V)$ by an onsite Coulomb interaction $U$. It means that a resonant transmission along the second channel takes place at a higher voltage than resonant transmission along the first channel.

According to the wide-band limit the tunneling flow takes the form

$Q_{\mathrm{LR}}(j)=\frac{1}{\pi \hbar} \int_{E_{\mathrm{F}}}^{E_{\mathrm{F}}+e V} \mathrm{~d} E T_{j}(E, V)$,

where the transmission function for the $j$ th tunneling channel reads

$T_{j}(E, V)=\frac{\Gamma_{\mathrm{L}} \Gamma_{\mathrm{R}}}{\left|E-\varepsilon(V)-j U+i\left(\Gamma_{\mathrm{L}}+\Gamma_{\mathrm{R}}\right) / 2\right|^{2}}$.

The flow $Q_{\mathrm{LR}}(j)$ is identical to the integral inter-electrode electron transfer rate. But in contrast to the electrodemolecule hopping rates $\chi_{\mathrm{L}}(j)$ and $\chi_{\mathrm{R}}(j)$ (as well as the backward rates $\chi_{-\mathrm{L}}(j)$ and $\left.\chi_{-\mathrm{R}}(j)\right)$ which describe electron transfer in the second order with respect to the leadmolecule coupling, the quantity $Q_{\mathrm{LR}}(j)$ accounts for the

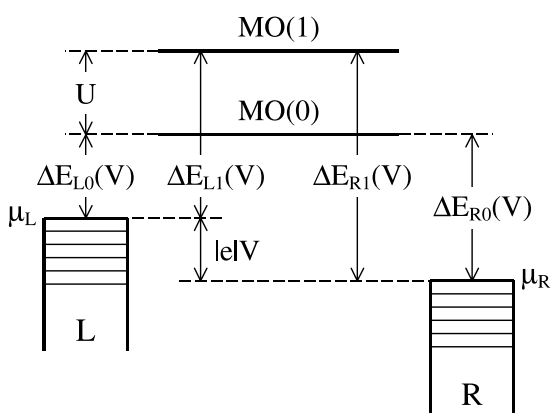

Fig. 3. Energy gaps appearing at the transmission of an electron through the considered single molecular level. The energy gaps $\Delta E_{\mathrm{L} 1}$ and $\Delta E_{\mathrm{R} 1}$ exceed the respective gaps $\Delta E_{\mathrm{L} 0}$ and $\Delta E_{\mathrm{R} 0}$ of the on-site Coulomb repulsion $U$ between the transferred electron and the electron already populating the molecule. 
transfer processes in an infinite order of lead-molecule coupling. However, if the level broadening $\left(\Gamma_{\mathrm{L}}+\Gamma_{\mathrm{R}}\right) / 2$ in the denominator of the transmission function (16) is ignored, one arrives at an expression which is of forthorder with respect to the lead-molecule coupling. This result can be also derived in the framework of a kinetic description of electron transfer [52] if one expands the respective relaxation operator with respect to the leadmolecule coupling.

\section{Current through the isolated molecular level}

Having the Eqs. (3) and (6)-(9) at hand we will concentrate our subsequent discussion on the stationary regime where the molecular level populations $P_{\uparrow}(t)$ and $P_{\downarrow}(t)$ become time-independent. Moreover, in the absence of a magnetic field we may set $P_{\uparrow}(t)=P_{\downarrow}(t) \equiv P(t)$ which in turn leads to the following expression for the stationary current

$I=I_{\text {seq }}+I_{\mathrm{dir}}, \quad I_{\mathrm{seq}}=\sum_{j=0,1} I_{\mathrm{seq}}(j), \quad I_{\mathrm{dir}}=\sum_{j=0,1} I_{\mathrm{dir}}(j)$.

The overall current consists of a part related to sequential charge transmission and a part related to a direct electron transfer. Moreover, each contribution is composed of two transmission channels related to the neutral molecule $(j=0)$ and to the singly charged molecule $(j=1)$. The sequential component of the current moving through the $j$ th channel reads

$$
\begin{aligned}
I_{\text {seq }}(j)= & I_{0} 2 \pi \Gamma_{\mathrm{L}}\left[n\left(\Delta E_{\mathrm{L} j}(V)\right) P^{j}(1-P)^{2-j}\right. \\
& \left.\left.-\left(1-n\left(\Delta E_{\mathrm{L} j}(V)\right)\right) P^{j+1}(1-P)^{1-j}\right)\right]
\end{aligned}
$$

whereas the related direct current component takes the form

$I_{\mathrm{dir}}(j)=I_{\mathrm{L}}(j)(j+1) P^{j}(1-P)^{2-j}$.

Here

$$
\begin{aligned}
I_{\mathrm{L}}(j)= & 2 I_{0} \frac{\Gamma_{\mathrm{L}} \Gamma_{\mathrm{R}}}{\Gamma_{\mathrm{L}}+\Gamma_{\mathrm{R}}}\left[\arctan \left(\frac{2 \Delta E_{\mathrm{R} j}(V)}{\Gamma_{\mathrm{L}}+\Gamma_{\mathrm{R}}}\right)\right. \\
& \left.-\arctan \left(\frac{2 \Delta E_{\mathrm{L} j}(V)}{\Gamma_{\mathrm{L}}+\Gamma_{\mathrm{R}}}\right)\right]
\end{aligned}
$$

is the tunneling current following from the Landauer scattering theory, applied to the case of a single molecular level. Note here also the introduction of $I_{0}=(|e|)$ $\pi \hbar) \times(1 \mathrm{eV}) \approx 80 \mu \mathrm{A}$.

The molecular single-electron population $P$ appearing in the Eqs. (18) and (19) follows as the stationary solution of Eq. (9), i.e. with $P_{\uparrow}(t)=P_{\downarrow}(t) \equiv P(t)=P$

$$
\begin{aligned}
& P=S /(1+S), \\
& S=\left[\sqrt{(A(0)-B(1))^{2}+4 B(0) A(1)}-(A(0)-B(1))\right] /(2 A(1))
\end{aligned}
$$

with
$A(j)=h\left(\chi_{-\mathrm{L}}(j)+\chi_{-\mathrm{R}}(j)\right), \quad B(j)=h\left(\chi_{\mathrm{L}}(j)+\chi_{\mathrm{R}}(j)\right)$.

\section{Results and discussion}

In the following we present a detailed discussion of the Eqs. (17)-(21). Main attention is focused on the interplay of the sequential and the direct transmission routes responsible for the formation of the total current (17). The main result of the present consideration is the fact that inelastic hopping processes not only form the sequential route for a charge transmission through the molecule but also control the tunneling route. Such a control occurs via the kinetic charging of the molecule caused just by the forward and backward hopping processes. Therefore, charge transmission takes place along two channels related to the neutral $(j=0)$ and the singly charged $(j=1)$ state of the molecule [53]. Transmission along the first channel corresponds to a pure single-electron transfer. At the same time, a singleelectron transmission along the second channel includes the Coulomb interaction between the incoming electron and the electron already captured by the molecule in the course of the hopping process. The resonant transmission along the $j$ th channel is switched on if $|V| \geqslant V_{r}(j)$ $(r=\mathrm{L}, \mathrm{R})$, where $V_{r}(j)$ is the resonant voltage. Its actual value is determined by the condition $\Delta E_{r j}(V)=0$. As it follows from Eq. (14) the energy gap $\Delta E_{\mathrm{L} j}(V)$ vanishes only at positive voltages. This yields

$V_{\mathrm{L}}(j)=(\Delta E(0)+j U) / \eta|e|$.

In contrast, the energy gap $\Delta E_{\mathrm{R} j}(V)$ only vanishes for negative voltages and, thus, we have

$V_{\mathrm{R}}(j)=(\Delta E(0)+j U) /(1-\eta)|e|$.

The difference between the voltages $V_{\mathrm{L}}(j)$ and $V_{\mathrm{R}}(j)$ is completely determined by the voltage division factor $\eta$. In the framework of the standard scattering theory of electron transmission it is just this factor that determines the electrostatic profile as well as the asymmetry in the $I-$ $V$ characteristics of the molecule $[1,3,49]$. Actually, the Landauer expression (20) is symmetric with respect to an interchange of the width parameters (11). Thus, the asymmetry is only associated to the energy gaps (14) which are identical if $\eta=0.5$. Therefore, at $\eta=0.5$ the $I-V$ characteristics displays the symmetry condition $I_{\mathrm{L}}(j ; V)=-I_{\mathrm{L}}(j ;-V)$, either for $\Gamma_{\mathrm{L}}=\Gamma_{\mathrm{R}}$ or for $\Gamma_{\mathrm{L}} \neq \Gamma_{\mathrm{R}}$. Our unified description shows that a current asymmetry can appear even at a symmetric electrostatic profile, provided that the hopping processes between the molecule and the electrodes are asymmetric so that $\Gamma_{\mathrm{L}} \neq \Gamma_{\mathrm{R}}$. To illuminate this result in we assume a symmetric profile with respect to the leads and set $\eta=0.5$, what results in $V_{\mathrm{R}}(j)=V_{\mathrm{L}}(j)$. Therefore, the resonant transmission regimes are switched on at symmetric voltage biases, $V=$ $V_{\mathrm{L}}(j)$ and $V=-V_{\mathrm{R}}(j)$.

As an example, let us consider the direct current through the first transmission channel. Fig. 4 shows that a unified description as well as the scattering theory leads to identical 

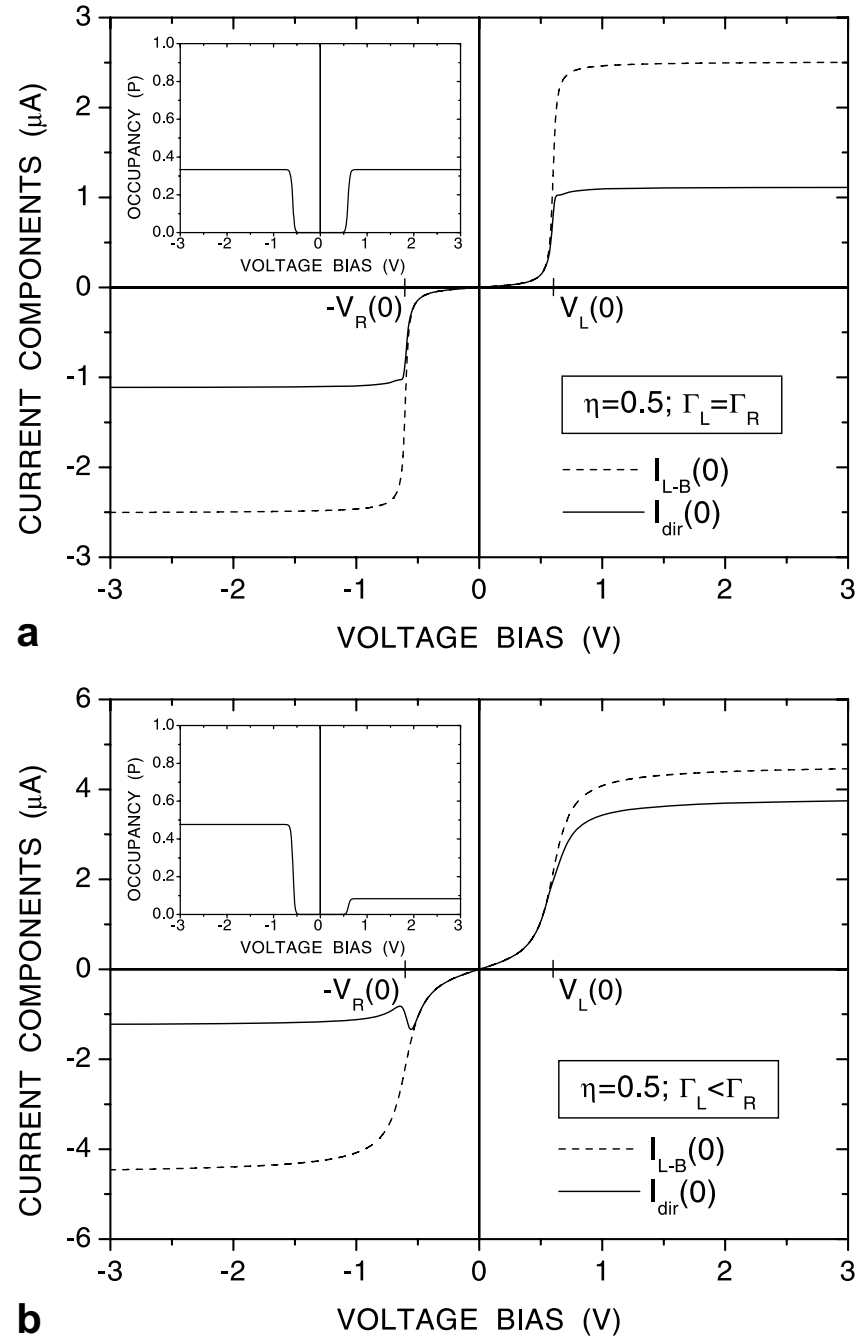

Fig. 4. $I-V$ characteristics of the molecule for a voltage division factor $\eta=1 / 2$ and for the direct electron transmission through the single molecular level as well as along the first transmission channel $(j=0)$. If the transmission is described in the framework of the Landauer scattering approach, Eq. (20), the current is symmetric at (a) $\Gamma_{\mathrm{L}}=\Gamma_{\mathrm{R}}$ and (b) $\Gamma_{\mathrm{L}} \neq \Gamma_{\mathrm{R}}$. The unified description of the current results in a direct part which is modified by a kinetic recharging of the molecule. The calculations have been done for $\Delta E(0)=0.3 \mathrm{eV}, U=5 \mathrm{eV}, k_{\mathrm{B}} T=0.01 \mathrm{eV}$ and (a) $\Gamma_{\mathrm{L}}=\Gamma_{\mathrm{R}}=0.01 \mathrm{eV}$, and (b) $\Gamma_{\mathrm{L}}=0.01 \mathrm{eV}, \Gamma_{\mathrm{R}}=0.1 \mathrm{eV}$.

results only in the pre-resonant voltage regions $0 \leqslant V<$ $V_{\mathrm{L}}(0)$ and $0 \geqslant V>-V_{\mathrm{R}}(0)$. In these regions, the electron hopping transfer from the left (right) electrode into the molecule appears as a thermally activation process with positive energy gaps $\Delta E_{\mathrm{L} 0}(V)$ and $\Delta E_{\mathrm{R} 0}(V)$ (cf. Figs. 2 and 3). Therefore, in the pre-resonant regions, the hopping transfer results in a small population of the molecule by the transferred electrons only, i.e. $P=P(V) \approx 0$. It then follows that $I_{\text {dir }}(0) \approx I_{\mathrm{L}}(0)$. The situation changes drastically when $V \geqslant V_{\mathrm{L}}(0)\left(V \leqslant-V_{\mathrm{R}}(0)\right)$, where a resonant electron transfer through the molecule becomes possible. Now, the energy gap $\Delta E_{\mathrm{L} 0}(V)\left(\Delta E_{\mathrm{R} 0}(V)\right)$ becomes negative and the moleculelead hopping transfer does not require any thermal activation (cf. Fig. 2). This results in a sudden rise of the population of the molecule by the transferred electrons.
Eq. (21) shows that in the post-resonant regions the singleelectron populations reduce to

$$
\begin{aligned}
& P=P(V>0)=\Gamma_{\mathrm{L}} /\left(2 \Gamma_{\mathrm{L}}+\Gamma_{\mathrm{R}}\right), \quad\left(V_{\mathrm{L}}(0) \leqslant V \leqslant V_{\mathrm{L}}(1)\right), \\
& P=P(V<0)=\Gamma_{\mathrm{R}} /\left(\Gamma_{\mathrm{L}}+2 \Gamma_{\mathrm{R}}\right), \quad\left(-V_{\mathrm{R}}(0) \geqslant V \geqslant-V_{\mathrm{R}}(1)\right) .
\end{aligned}
$$

If the molecule is symmetrically coupled to both leads the width parameters coincide $\left(\Gamma_{\mathrm{L}}=\Gamma_{\mathrm{R}}\right)$ and the population $P=P(V)$ becomes an even function of $V$, cf. the insert in Fig. 4a), i.e. we obtain $I_{\text {dir }}(0 ; V)=-I_{\text {dir }}(0 ;-V)$. If, however, $\Gamma_{\mathrm{L}} \neq \Gamma_{\mathrm{R}}$, then the hopping transfer becomes asymmetric. It results in an asymmetric transient population of the molecule by the transferred electrons (note the inequality $P(V)<P(-V)$ which is valid at $0 \leqslant V<V_{\mathrm{L}}(0)$ and $0 \geqslant-V>V_{\mathrm{R}}(0)$, cf. also the insert in Fig. 4b). Consequently, the direct current is also asymmetric. Fig. $4 \mathrm{~b}$ demonstrates this behavior for $\Gamma_{\mathrm{L}}<\Gamma_{\mathrm{R}}$. In this case and in the voltage region $0 \leqslant V<V_{\mathrm{L}}(0)$ the transition of an electron from the molecule to the right electrode is much faster than the transfer of an electron from the left electrode into the molecule. If, however, $0 \geqslant V>-V_{\mathrm{R}}(0)$ the reverse behavior takes place, i.e. the transition of an electron from the molecule into the left electrode is less effective than the transfer of an electron from the right electrode into the molecule. Just these asymmetric hopping transitions are responsible for the above mentioned differences between the single-electron populations at $0 \leqslant V<$ $V_{\mathrm{L}}(0)$ and at $0 \geqslant V>-V_{\mathrm{R}}(0)$. The nonsymmetric increase of the single-electron population results in an asymmetry of the current at $V>0$ and $V<0$ and, thus, in the appearance of a rectification effect. However, in the given case the rectification is not caused by a nonsymmetric shift of molecular level under the applied voltage (such a shift is associated with a voltage division factor $\eta$ if only the latter differs from 0.5). Rectification appears even at $\eta=0.5$. It is determined by nonsymmetric kinetic processes in the LMR-device caused nonsymmetric charge hopping between the molecule and each electrode. Therefore, one can say about specific kinetic rectification. (Here, and in what follows we illustrate the theoretical results choosing the parameters at which the calculated currents are in the $\mu \mathrm{A}$ range. Despite this seems high values for a single molecule, nevertheless, such currents are observed for small organic molecules $[8,48,54,55]$.)

Fig. $4 \mathrm{~b}$ shows the $I-V$ characteristics for a direct electron transfer and a restriction to the first transmission channel only. The complete direct electron transfer, however, involves two transmission channels which are related to the charged state of the molecule. The efficiency of each channel is determined by the strength of the Coulomb interaction between the extra electrons occupying the molecule in the course of the transmission process. As an example Fig. 5 displays the direct current in the voltage region $[-3,+3] \mathrm{V}$ and for two parameters of the Coulomb interaction, $U=2 \mathrm{eV}$ and $U=0.5 \mathrm{eV}$. A comparison with Fig. $4 \mathrm{~b}$ does not indicate any pronounced difference 


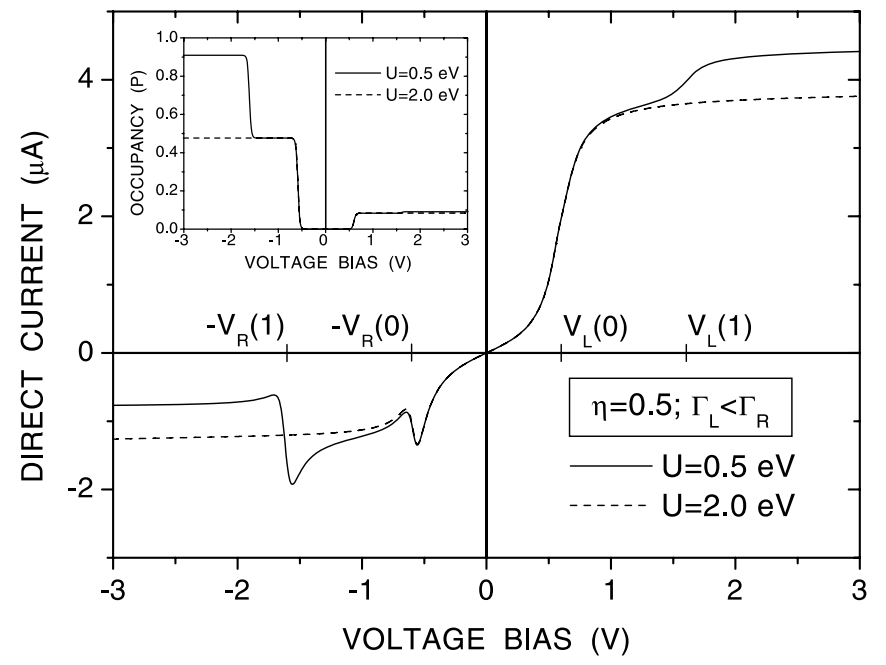

Fig. 5. The current component related to the direct transfer versus the applied voltage $V$. The modification (via a kinetic recharging of the molecule) is especially large in the post-resonant voltage regions $V>V_{\mathrm{L}}(0)$ and $V<-V_{\mathrm{R}}(0)$ where a resonant opening of the two transmission channels occurs. The first channel $(j=0)$ and the second channel $(j=1)$ are switched on at $\left.V=V_{\mathrm{L}}(0)\left(V=-V_{\mathrm{R}}(0)\right)\right)$ and $V=V_{\mathrm{L}}(1)\left(V=-V_{\mathrm{R}}(1)\right)$, respectively. The sawtooth shape of the $I-V$ characteristics at a negative current (at $U=0.5 \mathrm{eV}$ ) is dictated by a substantial current drop caused by the large and sudden increase of the molecular level population $P$ at $V=-V_{\mathrm{R}}(0)$ and $V=-V_{\mathrm{R}}(1)$. A similar increase of $P$ is insignificant at $V>0$, see the insert. This effect is caused by an asymmetric molecule-lead coupling $\Gamma_{\mathrm{L}} \neq \Gamma_{\mathrm{R}}$. Here, the case $\Gamma_{\mathrm{L}}=0.1 \Gamma_{\mathrm{R}}$ is presented. At a large value of Coulomb repulsion between the transferred electrons occupying the molecule, the second peak is positioned at $V<-3 \mathrm{~V}$. The calculations are based on Eq. (19) and for the parameter set $\Delta E(0)=0.3 \mathrm{eV}, k_{\mathrm{B}} T=$ $0.01 \mathrm{eV} ; \Gamma_{\mathrm{L}}=0.01 \mathrm{eV}, \Gamma_{\mathrm{R}}=0.1 \mathrm{eV}$.

between the $I-V$ characteristics derived at $U=5 \mathrm{eV}$ (Fig. 4b) and $U=2 \mathrm{eV}$ (Fig. 5). In both cases, only a single resonance region appears. This indicates that the second transmission channel contributes only weakly. Such a situation changes if the Coulomb interaction becomes comparable to the (unbiased) energy gap $\Delta E(0)$. Now, the new resonant voltages, $V_{\mathrm{L}}(1)$ and $V_{\mathrm{R}}(1)$, are also positioned in the voltage region $[-3,+3] \mathrm{V}$ where already the resonant voltages $V_{\mathrm{L}}(0)$ and $V_{\mathrm{R}}(0)$ are located. If $V=V_{\mathrm{L}}(1)$ and $V=-V_{\mathrm{R}}(1)$ a new sudden increase of single-electron populations takes place where the latter are given as

$P=P(V>0)=\Gamma_{\mathrm{L}} /\left(\Gamma_{\mathrm{L}}+\Gamma_{\mathrm{R}}\right), \quad\left(V \geqslant V_{\mathrm{L}}(1)\right)$,

$P=P(V<0)=\Gamma_{\mathrm{R}} /\left(\Gamma_{\mathrm{L}}+\Gamma_{\mathrm{R}}\right), \quad\left(V \leqslant-V_{\mathrm{R}}(1)\right)$.

In the case $\Gamma_{\mathrm{L}}=0.1 \Gamma_{\mathrm{R}}$ under consideration, these populations exceed those given by Eq. (25), especially at $V<0$. Each sudden increase of $P$ modifies the direct current component. In Fig. 4, the $I-V$ characteristics have been calculated for $\Delta E(0)=0.3 \mathrm{eV}$ and $U=$ $0.5 \mathrm{eV}$. Consequently, the resonant regimes for the first and the second transmission channels are switched on at $V=V_{\mathrm{L}}(0)=0.6 \mathrm{~V}\left(V=-V_{\mathrm{R}}(0)=-0.6 \mathrm{~V}\right)$ and $V=$ $V_{\mathrm{L}}(1)=1.6 \mathrm{~V}\left(V=-V_{\mathrm{R}}(1)=-1.6 \mathrm{~V}\right)$, respectively. This can be clearly seen at negative voltages where the sudden increase of the molecular population is much stronger as that for the similar increase at positive voltages. In the negative voltage region, each increase of the population is accompanied by the appearance of a current peak (cf. the insert of Fig. 5) and thus by a respective negative differential resistance. The formation of the peaks is completely correlated with a sudden increase of the MO's electronic population $P$. If the resonant regime is reached it changes from $P=0$ up to $P=1 / 2.1 \approx 0.48$ for the first transmission channel and from $P \approx 0.46$ up to $P=1 /$ $1.1 \approx 0.90$ for the second (cf. the insert to Fig. $5 \mathrm{a}$ as well as Eqs. (25) and (26)). At the same time, the absence of such peaks in the region of positive voltages is dictated by a small sudden rise of the populations from $P=0$ up to $P=0.1 / 1.2 \approx 0.08$ and from $P \approx 0.08$ up to $P=0.1 / 1.1 \approx 0.09$ for the first and the second transmission channels, respectively. Such a small change of the populations is compensated by a monotonous increase of the tunneling component (20) with $V$.

Next, let us consider the formation of the sequential current component (18). The related $I-V$ characteristics display certain steps as shown in Fig. 6 which follows from the fact that up to room temperatures the distribution functions (13) are close to the unit-step functions. Therefore, we obtain

$I_{\text {seq }} \simeq 0,\left(0 \leqslant V<V_{\mathrm{L}}(0), 0>V \geqslant-V_{\mathrm{R}}(0)\right)$.

The first threshold occurs at $V=V_{\mathrm{L}}(0)$ (or $V=-V_{\mathrm{R}}(0)$ ) and reflects the resonant opening of the first transmission channel. Apparently, the sequential transfer route starts with an electron jump from a lead band-level into the neutral molecule. Thereafter, the electron jumps further from the molecule into an empty band level of another electrode (cf. the scheme in Fig. 2). It follows from the Eqs. (17), (18) and (25) that the resulting current is given by the expression

$$
\begin{array}{ll}
I_{\text {seq }}=I_{0} 2 \pi \Gamma_{\mathrm{L}} \Gamma_{\mathrm{R}} /\left(2 \Gamma_{\mathrm{L}}+\Gamma_{\mathrm{R}}\right), & \left(V_{\mathrm{L}}(0) \leqslant V \leqslant V_{\mathrm{L}}(1)\right), \\
I_{\text {seq }}=I_{0} 2 \pi \Gamma_{\mathrm{L}} \Gamma_{\mathrm{R}} /\left(\Gamma_{\mathrm{L}}+2 \Gamma_{\mathrm{R}}\right), & \left(-V_{\mathrm{R}}(0) \geqslant V \geqslant-V_{\mathrm{R}}(1)\right) .
\end{array}
$$

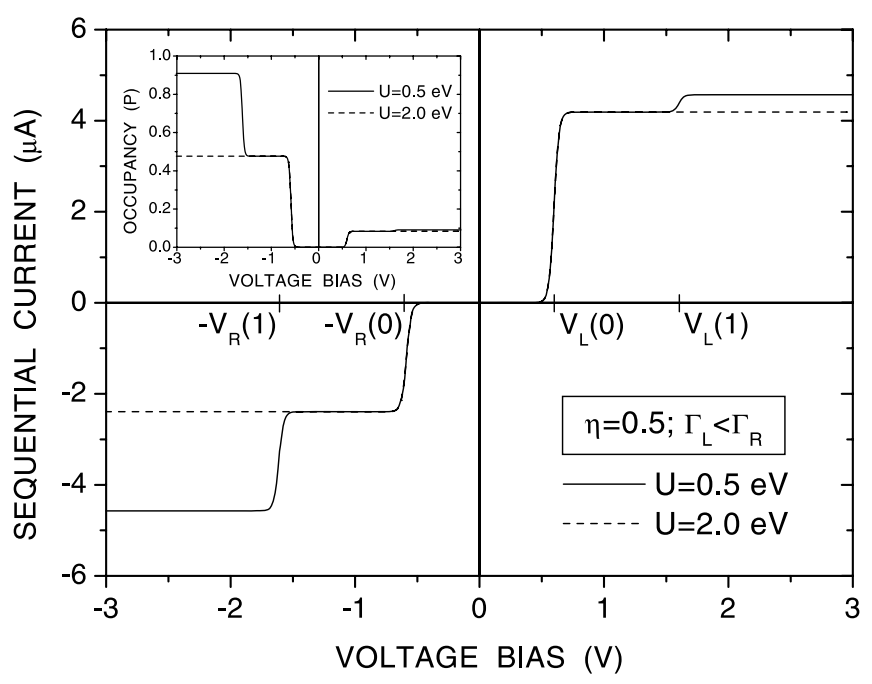

Fig. 6. Step-like behavior of the sequential current component. The calculations are based on Eq. (18) and the same parameters are used as in Fig. 4. The steps result from a switching-on of the resonant transmission regimes at $V=V_{\mathrm{L}}(0)\left(V=-V_{\mathrm{R}}(0)\right)$ and $V=V_{\mathrm{L}}(1)\left(V=-V_{\mathrm{R}}(1)\right)$. 
The second threshold in the $I-V$ characteristics appears at $V=V_{\mathrm{L}}(1)$ (or $V=-V_{\mathrm{R}}(1)$ ) corresponding to the resonant opening of the second transmission channel where the electron jumps into an already singly charged molecule. According to the Coulomb interaction of the incoming electron with the electron already present in the molecule the second resonance is shifted to larger values of the applied voltage. Afterwards, one of the two electrons localized at the molecule jumps back to another electrode. In such a case where two transmission channels are involved in the current formation one obtains

$$
\begin{aligned}
& I_{\text {seq }}=I_{0} 2 \pi \Gamma_{\mathrm{L}} \Gamma_{\mathrm{R}} /\left(\Gamma_{\mathrm{L}}+\Gamma_{\mathrm{R}}\right), \quad\left(V \geqslant V_{\mathrm{L}}(1)\right), \\
& I_{\text {seq }}=I_{0} 2 \pi \Gamma_{\mathrm{L}} \Gamma_{\mathrm{R}} /\left(\Gamma_{\mathrm{L}}+\Gamma_{\mathrm{R}}\right), \quad\left(V \leqslant-V_{\mathrm{R}}(1)\right) .
\end{aligned}
$$

This expression indicates that in the case where both transmission channels participate in the formation of the sequential transfer route the rectification disappears. This is in a strong contrast to the behavior of the current related to the direct transmission route, see the behavior in Fig. 5 vs. Fig. 6).

Note that in the pre-resonant regions $0 \leqslant V<V_{\mathrm{L}}(0)$ and $0 \geqslant V>-V_{\mathrm{R}}(0)$ the current component of the direct transfer exceeds the component related to the sequential transfer (the latter is equal zero). In the post-resonant region $V>V_{\mathrm{L}}(0)$, the transmission along both routes results in nearly identical contributions to the total current, while in the post-resonant region $V<-V_{\mathrm{R}}(0)$ the sequential current component exceeds the direct one. Accordingly, the actual $I-V$ characteristics of a molecule can rather well be estimated if the sequential and the direct transfer routes are taken into consideration.

\section{Conclusions}

In the present communication we analyzed the electron transfer through a single molecule attached to two electrodes. Our unified description of charge transmission developed earlier combined with the restriction to a single molecular level to be occupied allowed us to describe the tunneling and sequential route of transfer in using a minimal set of parameters. These are: the width parameters $\Gamma_{\mathrm{L}}$ and $\Gamma_{\mathrm{R}}$, the voltage division factor $\eta$, the unbiased energy gap $\Delta E(0)$, and the strength of on-site Coulomb repulsion $U$. It could be shown that the sequential and the tunneling route of electron transfer occur through two transmission channels associated with the neutral as well as with the singly charged molecule. Both mentioned molecular states as well as the doubly charged state comprise the set of states possible for a single level.

Transitions between these charging states are determined by single-electron electrode-molecule/moleculeelectrode hopping processes. If a stationary regime of the current is established, then the weight of each molecular charging state is determined by the relation between these hopping rates. Noting that a tunneling transfer does not change the molecular charge, one realizes that just the inelastic sequential mechanism of charge transmission becomes responsible for a kinetic recharge of the molecule. As a result, the tunneling occurs against the background of kinetically controlled molecular charging states. It is helpful to analyze the correlation between the single-electron MO-population $P$ and the $I-V$ characteristics (compare the insert and the current plot in each of the Figs. 4-6). Accordingly, the tunneling component of the current is modified by inelastic hopping processes. To underline this fact we used the term "direct current" instead of the term "tunneling current". Note that a formation of the direct current is associated with both, the virtual and the real occupation of the empty or singly populated MO by the transferred electrons.

Electron transmission accompanied by a virtual population of the MO is similar to the co-tunneling process known from mesoscopic physics [50,51]. The efficiency of the direct tunneling strongly depends on the kinetic population of the MO by the extra electrons which really populate the MO according to the incoherent hopping process. Thus, direct tunneling can be referred to as co-tunneling which is controlled by a kinetic charging of the molecule. Direct tunneling also appears in a resonant regime where the molecular recharge (by the electrode-molecule/electrode-molecule electron hoppings) becomes significant. The expression for the direct current component, Eq. (19) clearly displays the noted control: a direct current component is formed owing to a tunnelling transmission (with the virtual population of the $\mathrm{MO}$ ), and it is characterized by a tunneling current $I_{\mathrm{L}}(j)$, Eq. (20). However, the probability $W_{j}$ (where $\left.W_{0}=(1-P)^{2}, W_{1}=P(1-P)\right)$ to incorporate the $j$ th tunneling pathway into the formation of the direct current is exclusively defined by incoherent hopping processes responsible for a real population of the MO. It follows from the fact that the single-electron population $P$ only depends on the relation between the hopping rates (cf. Eqs. (21) and (22)).

In the wide band limit, the hopping rates are expressed through the width parameters $\Gamma_{\mathrm{L}}$ and $\Gamma_{\mathrm{R}}$ as well as the electronic distribution functions, Eq. (12). The latter are defined by the voltage controlled energy gaps (cf. Eq. (14) and Fig. 3). When one of the gap becomes negative, a resonant regime of transmission is switched-on changing substantially the character of the jumps. Resonant jumps lead to a step-like behavior of the molecular level populations $P_{\uparrow}=P_{\downarrow}=P$ and thus to a molecular recharging. It could be demonstrated that at an asymmetric coupling of the molecule to each electrode $\left(\Gamma_{\mathrm{L}} \neq \Gamma_{\mathrm{R}}\right)$, a kinetic change of the molecular charging state leads to a specific (kinetic) rectification of the tunneling transmission even though the electrostatic profile along the molecule remains identical at positive and negative voltages. A LMR-device with a symmetric potential profile in the inter-electrode region $(\eta=0.5)$ and, simultaneously, with asymmetric contacts $\left(\Gamma_{\mathrm{L}} \neq \Gamma_{\mathrm{R}}\right)$ can be fabricated utilizing a symmetric molecule with nonidentical terminal atoms. To organize a transmission regime with a single active molecular level becomes, 
probably, possible if the molecule includes a metallic ion (e.g. ion $\mathrm{Cu}^{2+}$ ) the atomic orbitals of which are able to create a well separated LUMO (HOMO) with other atoms. In the present studies, we analyzed the current asymmetry caused by the asymmetry in the hopping molecular-electrodes processes. If, additionally, an asymmetric voltage distribution along the molecule occurs the factor $\eta$ differs from 0.5. In this case, resonant voltages (23) and (24) do not coincide and thus a resonant switching on the transmission channels occurs at different positive and negative voltages. It means that the current asymmetry is now achieved owing to both the asymmetric shift of the active molecular level (caused by the applied voltage) and the asymmetric molecule-electrode hopping processes.

The results based on the unified description of electron transfer process show that in the resonant regime of electron transmission, the tunneling component of the current becomes strongly modified by the hopping processes via the alteration of the charging state of the molecule. This conclusion has to be considered as the main result of the present studies.

\section{Acknowledgements}

One of us (E.G.P.) gratefully acknowledge generous support by the Alexander von Humboldt Foundation. The work was also supported in part by the NATO scientific program (Ya.R.Z.).

\section{References}

[1] W. Tian, S. Datta, S. Hong, R. Reifenberger, J.I. Henderson, C.P. Kubiak, J. Chem. Phys. 109 (1998) 2874.

[2] M. Reed, Proc. IEEE 87 (1999) 652.

[3] R.M. Metzger, Acc. Chem. Res. 32 (1999) 950.

[4] C. Kergueris, J.-P. Bourgoin, S. Palacin, D. Esteve, C. Urbina, M. Magoga, C. Joachim, Phys. Rev. B 59 (1999) 12505.

[5] A. Nitzan, Ann. Rev. Phys. Chem. 52 (2001) 681.

[6] P. Hänggi, M. Ratner, S. Yaliraki, Chem. Phys. 281 (2002) 111.

[7] F. Zahid, M. Paulsson, S. Datta, in: Morkos (Ed.), Edvanced Semiconductors and Organic Nano-techniques, Acad. Press, New York, 2003, p. 41 (Chapter 2).

[8] S. Datta, Nanotechnology 15 (2004) S433.

[9] J.G. Kushmerick, C.M. Whitaker, S.K. Pollack, T.L. Schull, R. Shashidhar, Nanotechnology 15 (2004) S489.

[10] A.W. Ghosh, P.S. Damle, S. Datta, A. Nitzan, MRS Bulletin (June) (2004) 391.

[11] J.G. Kushmerick, D.B. Holt, J.C. Yang, J. Naciri, M.H. Moore, R. Shashidhar, Phys. Rev. Lett. 89 (2002) 086802.

[12] A. Nitzan, M.A. Ratner, Science 300 (2003) 1384.

[13] S. Datta, Electronic Transport in Mesoscopic Systems, University Press, Cambridge, 1995.

[14] S. Kohler, J. Lehmann, P. Hänggi, Phys. Rep. 406 (2005) 379.

[15] S. Camalet, J. Lehmann, S. Kohler, P. Hänggi, Phys. Rev. Lett. 90 (2003) 210602;

S. Camalet, S. Kohler, P. Hänggi, Phys. Rev. B 70 (2004) 155326.

[16] M. Cizek, M. Thoss, W. Domcke, Phys. Rev. B 70 (2004) 125406.

[17] J. Lehmann, S. Kohler, V. May, P. Hänggi, J. Chem. Phys. 121 (2004) 2278.
[18] J. Reichert, R. Ochs, D. Beckmann, H.B. Weber, M. Mayor, H.v. Löneysen, Phys. Rev. Lett. 88 (2002) 176804

[19] S. Tikhodeev, M. Natario, K. Makoshi, T. Mii, Y. Ueba, Surf. Sci. 493 (2001) 63.

[20] M. Galperin, M.A. Ratner, A. Nitzan, J. Chem. Phys. 121 (2004) 11965.

[21] E.G. Petrov, V. May, P. Hänggi, Chem. Phys. 296 (2004) 251.

[22] E.G. Petrov, V. May, P. Hänggi, Chem. Phys. 319 (2005) 380.

[23] E.G. Petrov, I.S. Tolokh, A.A. Demidenko, V.V. Gorbach, Chem. Phys. 193 (1995) 237.

[24] E.G. Petrov, P. Hänggi, Phys. Rev. Lett. 86 (2001) 2862.

[25] A. Nitzan, J. Phys. Chem. A 105 (2001) 2677.

[26] A.M. Kuznetsov, J. Ulstrup, J. Chem. Phys. 116 (2002) 2149.

[27] V. Mujica, A.E. Roitberg, M.A. Ratner, J. Chem. Phys. 112 (2000) 6834.

[28] M.H. Hettler, H. Schoeller, W. Wenzel, Europ. Lett. 57 (4) (2002) 571.

[29] E.G. Petrov, V. May, P. Hänggi, Chem. Phys. 281 (2002) 211.

[30] A. Nitzan, M. Galperin, G.-L. Ingold, H. Grabert, J. Chem. Phys. 117 (2002) 10837.

[31] V. Mujica, A. Nitzan, S. Dutta, M. Ratner, C.P. Kubiak, J. Phys. Chem. B 107 (2003) 91.

[32] S.K. Pati, J. Chem. Phys. 118 (2003) 6529.

[33] F. Orellana, F. Claro, Phys. Rev. Lett. 90 (2003) 178302.

[34] G.C. Liang, A.W. Ghosh, M. Paulsson, S. Datta, Phys. Rev. B 69 (2004) 115302.

[35] W. Häussler, B. Kramer, J. Masek, Z. Phys. B 85 (1991) 435.

[36] A.L. Yeyati, A. Martin-Rodero, F. Flores, Phys. Rev. Lett. 71 (1993) 2991.

[37] A.M. Kuznetsov, J. Ulstrup, J. Electroanal. Chem. 564 (2004) 209.

[38] A. Mitra, I. Aleiner, A.J. Millis, Phys. Rev. B 69 (2004) 245302.

[39] C. Joachim, M. Ratner, PNAS 102 (2005) 8801.

[40] M. Galperin, A. Nitzan, M. Ratner, Phys. Rev. B 73 (2006) 045314.

[41] P.W. Anderson, Phys. Rev. 124 (1961) 41.

[42] The used Hubbard version of the Coulomb repulsion is valid for well localized MOs. For definite conditions, in particular, in the case of well separated extended molecular levels, the electron-electron interaction can be described by using a truncated form that is similar to the Hubbard-form [22].

[43] Y. Meir, N.S. Wingreen, P.A. Lee, Phys. Rev. Lett. 66 (1991) 3048.

[44] Y. Meir, N.S. Wingreen, P.A. Lee, Phys. Rev. Lett. 70 (1993) 2601.

[45] J. Lehmann, G.-L. Ingold, P. Hänggi, Chem. Phys. 281 (2002) 199.

[46] If only a pure electronic Hamiltonian (1) is used for a description of electron transfer process then one can obtain a coarse-grained description only.

[47] P.S. Damle, A.W. Ghosh, S. Datta, Phys. Rev. B 64 (2001) 201403.

[48] H.B. Weber, J. Reichert, F. Weigend, R. Ochs, D. Beckmann, M. Mayor, R. Ahlrichs, H.v. Löhneysen, Chem. Phys. 281 (2002) 113.

[49] E.G. Petrov, Low Temp. Phys. 31 (2005) 338.

[50] D.V. Averin, Yu. V. Nazarov, Phys. Rev. Lett. 65 (1990) 2446.

[51] L.I. Glazman, M. Pustilnik, in: H. Bouchiat, Y. Gefen, S. Gueron, G. Montambaux, J. Dalibard (Eds.), Nanophysics: Coherence and Transport, Elsevier, Amsterdam, 2005, p. 427.

[52] M. Sparpaglione, S. Mukamel, J. Chem. Phys. 88 (1988) 3263.

[53] A doubly charged molecule does not transmit the extra electron between the leads. This is due to the fact that within the given voltage region only a single $\mathrm{MO}$ is assumed to be involved in the transmission process. Since the MO is already completely filled by two extra electrons, then, the third electron cannot be captured by this MO and cannot create a threefold charged virtual or real state with the participation of this single MO.

[54] X. Xiao, B. Xu, N.J. Tao, Nano Lett. 4 (2004) 267.

[55] T. Rakshit, G.-C. Liang, A.W. Ghosh, S. Datta, Nano Lett. 4 (2004) 1803. 